\title{
Transmission and reflection properties of terahertz fractal metamaterials
}

\author{
Malureanu, Radu; Lavrinenko, Andrei; Cooke, David; Jepsen, Peter Uhd; Xiao, S.; Zhou, L.
}

Published in:

2010 Conference on Lasers and Electro-Optics (CLEO) and Quantum Electronics and Laser Science Conference (QELS)

Publication date:

2010

Document Version

Publisher's PDF, also known as Version of record

Link back to DTU Orbit

Citation (APA):

Malureanu, R., Lavrinenko, A., Cooke, D., Jepsen, P. U., Xiao, S., \& Zhou, L. (2010). Transmission and reflection properties of terahertz fractal metamaterials. In 2010 Conference on Lasers and Electro-Optics (CLEO) and Quantum Electronics and Laser Science Conference (QELS) (pp. 1-2). IEEE.

\section{General rights}

Copyright and moral rights for the publications made accessible in the public portal are retained by the authors and/or other copyright owners and it is a condition of accessing publications that users recognise and abide by the legal requirements associated with these rights.

- Users may download and print one copy of any publication from the public portal for the purpose of private study or research.

- You may not further distribute the material or use it for any profit-making activity or commercial gain

- You may freely distribute the URL identifying the publication in the public portal 


\title{
Transmission and Reflection Properties of Terahertz Fractal Metamaterials
}

\author{
Radu Malureanu, Andrei Lavrinenko, David G. Cooke, and Peter Uhd Jepsen \\ DTU Fotonik, Technical University of Denmark, DK-2800 Kongens Lyngby, Denmark \\ puje@fotonik.dtu.dk \\ S. Xiao, L. Zhou \\ Fudan University - Physics Department, Shanghai 200433, China
}

\begin{abstract}
We use $\mathrm{THz}$ time-domain spectroscopy to investigate transmission and reflection properties of metallic fractal metamaterial structures. We observe loss of free-space energy at certain resonance frequencies, indicating excitation of surface modes of the metamaterial.

(C)2010 Optical Society of America

OCIS codes: (160.3918) Metamaterials; (300.6495) Spectroscopy, terahertz; (310.6628) Subwavelength structures, nanostructures
\end{abstract}

\section{Introduction}

Metamaterials (MTMs) are currently considered for extensive broadening of the functionalities of photonic components, for example in control over light polarization [1]. A tricky problem at $\mathrm{THz}$ frequencies is that no so many good designs have been proposed for polarization devices. We believe that planar fractal MTMs could be good generic candidates for the THz range, assuming relevant scaling of geometrical sizes. Such structures have been characterized previously [2,3], however, only in transmission. To gain further insight into the MTMs properties the transmission spectra (both amplitude and phase) should be complemented with the corresponding reflection data, for instance as it was done theoretically in the microwave region [4]. Here we report on fabrication and comprehensive characterization of a THz fractal MTM that show resonant transmission and reflection at certain frequencies within the $0.4-1.2 \mathrm{THz}$ range. A clear polarisation-dependent behaviour is monitored, and a region with enhanced losses at $1.05 \mathrm{THz}$ is identified. Such losses are possibly associated with the coupling to surface modes.

\section{Fabrication}

The structures (Fig 1. left) were fabricated on a high-resistivity $(>10 \mathrm{k} \Omega / \mathrm{cm})$ Si substrate. The substrate was coated with a $50 \mathrm{~nm}$ thin Au layer and then, using optical lithography, the structures were defined in polymer. The next process step involved electrochemical growth of $4 \mu \mathrm{m}$ of $\mathrm{Ni}$ and selective removal of the Au layer. The structures were afterwards removed from the substrate, thus obtaining free-standing metal films which are easier to characterise due to the lack of losses and FabryPerot resonances.
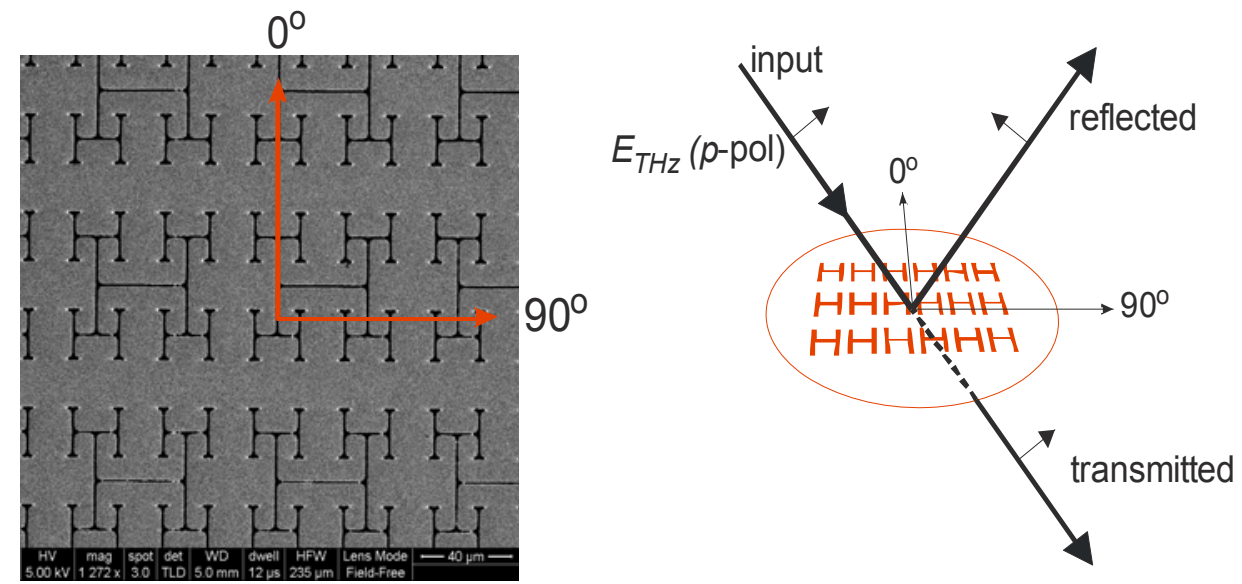

Fig. 1: (left) Microphotograph of the sample structure and (right) experimental geometry for the measurements, indicating the p-polarization of the $\mathrm{THz}$ light and the rotation angle of the reflection plane with respect to the structure.

\section{Measurements}


Transmission and reflection measurements were carried out with a standard $\mathrm{THz}$ time-domain spectroscopy (THz-TDS) system [5]. The sample geometry and the excitation angles are shown in Fig. 1. In the transmission experiments the $\mathrm{THz}$ light was incident on the sample at an angle of $20^{\circ}$, with identical transmission coefficient measured at normal incidence, indicating little influence of the transmission properties on the incidence angle. In reflection the incidence angle was fixed at 30 degrees by the experimental setup. We performed measurements at two different rotations of the sample with respect to the reflection plane $-0^{\circ}$ and $90^{\circ}$ as indicated in Fig. 1. In the transmission experiments the reference signal was recorded through an empty aperture, and in the reflection measurements, the reference signal was recorded with a plane, unstructured metal surface with a reflection coefficient of -1 . The amplitude of the measured reflection coefficient was difficult to measure in absolute terms due to slight variations of the surface profile of the sample. However, we estimate the uncertainty on the reflection amplitude to be approximately $10 \%$. Figure 2(a) shows the measured transmission amplitude and phase, and Fig. 2(b) shows the corresponding reflection amplitude and phase. At $0^{\circ}$ rotation of the sample we observe a transmission peak at $0.55 \mathrm{THz}$, and at $90^{\circ}$ rotation a slightly broader transmission peak at $1.0 \mathrm{THz}$. Both these resonances are also seen as pronounced dips in the reflection spectra. At each resonance we observe a characteristic phase jump of $\pi$ radians of the transmission spectra. And a smaller phase jump in the reflected signal.

The geometric air fraction of the surface is approximately 5\%. The structuring of the surface thus allows a significantly enhanced (up to $16 \mathrm{x}$ ) transmission at the resonances.

Interestingly, we observe a deep minimum $(\mathrm{t}=0.3 \%)$ in the transmission amplitude and a marked $\pi$ phase jump at $1.05 \mathrm{THz}$ through the sample at $0^{\circ}$ rotation. The minimum of the transmission amplitude is not accompanied as usual by an increase in the reflection coefficient, thus clearly indicating that energy is absorbed to excite a surface mode at this particular frequency. Further experiments, e.g. Attenuated Total Reflection (ATR) measurements, are being performed to support this conclusion.
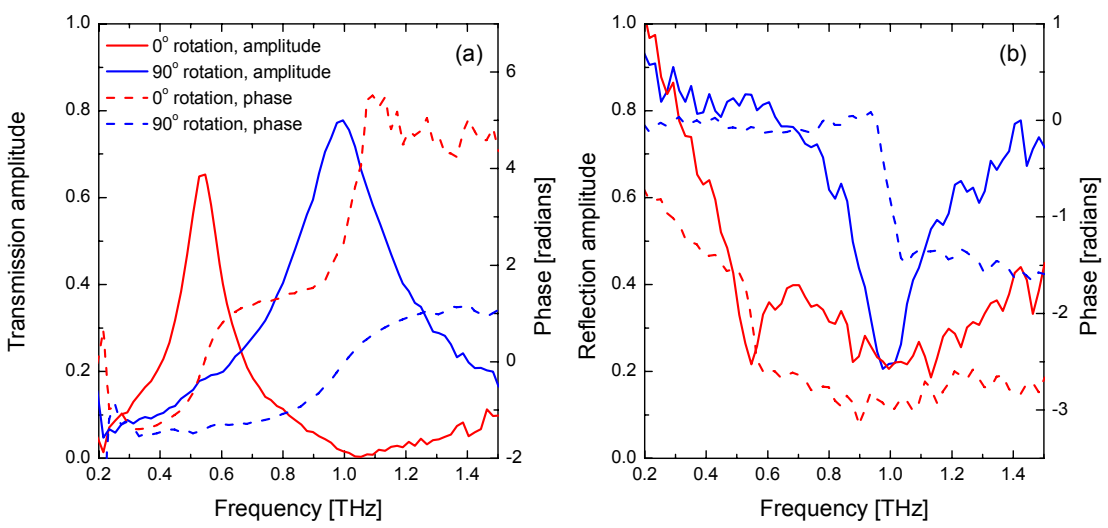

Fig. 2: (a) Transmission and (b) reflection coefficients (solid curves: amplitude, dashed curves: phase) with results for $0^{\circ}$ rotation of the sample shown in red and $90^{\circ}$ rotation shown in blue.

\section{Conclusions}

We have characterized the transmission and reflection properties of a $4-\mu \mathrm{m}$ thick free-standing metal film with a fractal metamaterial hole structure. For orthogonal incidence rotations the transmission is strongly enhanced near the frequencies of the first resonant currents in the fractal structure, in agreement with simulation results. In addition to the enhanced transmission, we observe a marked energy loss at a specific frequency for one of the sample orientations, indicating that energy is coupled with high efficiency from the free-space mode of the THz field to the structured surface.

[1] J. Hao, Q. Ren, Z. An, X. Huang, Z. Chen, M. Qiu and L. Zhou, “Optical metamaterial for polarization control,” Phys. Rev. A 80, 023807 (2009)

[2] G. Zhao, Y. Tian, H. Sun, C. Zhang, and G. Yang, "Multiband terahertz photonic band gaps of subwavelength planar fractals," Chin. Phys. Lett. 23, 1456-1458 (2006)

[3] F. Miyamaru, Y. Saito, M. W. Takeda, B. Hou, L. Liu, W. Wen, and P. Sheng, "Terahertz electric response of fractal metamaterial structures," Phys. Rev B 77, 045124 (2008)

[4] L. Zhou, C T Chan and P. Sheng, "Theoretical studies on the transmission and reflection properties of metallic planar fractals,” J. Phys. D: Appl. Phys. 37, 368-373 (2004)

[5] P. Uhd Jepsen, U. Møller, and H. Merbold, "Investigation of aqueous alcohol and sugar solutions with reflection terahertz time-domain spectroscopy,” Opt. Express 15, 14717-14737 (2007) 